\title{
Nondestructive diagnostics of soluble sugar, total nitrogen and their ratio of tomato leaves in greenhouse by polarized spectra-hyperspectral data fusion
}

\author{
Wenjing Zhu ${ }^{1,2^{*}}$, Jinyang $\mathrm{Li}^{1,2}$, Lin $\mathrm{Li}^{1,2}$, Aichen Wang ${ }^{1,2}$, Xinhua Wei ${ }^{1,2}$, Hanping Mao ${ }^{1,2}$ \\ (1. School of Agricultural Equipment Engineering, Jiangsu University, Zhenjiang 212013, China; \\ 2. Key Laboratory of Modern Agricultural Equipment and Technology, Ministry of Education, Jiangsu University, Zhenjiang 212013, China)
}

\begin{abstract}
Polarized spectra-hyperspectral data fusion technique was used to estimate the soluble sugar (SS), total nitrogen (N), and their ratio (SS/N), of greenhouse tomato leaves. Fresh tomato leaves of five different growth stages (seedling, flowering, initial fruiting, mid-fruiting and picking stage) and five different nitrogen treatments (severe stress $25 \%$, moderate stress $50 \%$, mild stress $75 \%$, normal $100 \%$, and excess $150 \%$ ) at every stage were collected for spectra acquisition and SS and $\mathrm{N}$ determination. Polarized reflectance spectra were acquired with a polarization reflectance spectrum spectro-goniophotometer system and four polarization degree features were extracted. Hyperspectral data were collected with a hyperspectral imaging system and four reflectance spectrum features and eight image features were extracted. Initially, models were built with polarization degree features, image features, and spectral features respectively. Linear and nonlinear fusion methods were comparatively used for modeling based on normalized data of the three sources. The results suggest that the performances of SS/N models are better than those of N and SS models, and the prediction capability of the Support Vector Machine (SVM) models of $\mathrm{N}$ and SS/N are superior to those obtained with single kind feature. This work indicates that the polarized spectrum-hyperspectral multidimensional information detecting method can feasibly judge the tomato nutrient stress conditions. Multi-features data fusion analysis technique can enhance the prediction accuracy of spectral diagnostics technology in precision agriculture.
\end{abstract}

Keywords: polarized spectra, hyperspectral, soluble sugar (SS), total nitrogen (N), data fusion, tomato leaf DOI: $10.25165 /$ j.ijabe.20201302.4280

Citation: Zhu W J, Li J Y, Li L, Wang A C, Wei X H, Mao H P. Nondestructive diagnostics of soluble sugar, total nitrogen and their ratio of tomato leaves in greenhouse by polarized spectra-hyperspectral data fusion. Int J Agric \& Biol Eng, 2020; 13(2): 189-197.

\section{Introduction}

Carbon $(\mathrm{C})$ and nitrogen $(\mathrm{N})$ are the basic constituent elements of organic matter; thus, quantitative monitoring of carbonaceous and nitrogenous substances in plants is an important research direction $^{[1,2]} . \quad \mathrm{N}$ is the main component of proteins, chlorophyll and nucleic acids, and is thus known as "the element of crop life" ${ }^{[3]}$. Different nutrient physiologic conditions, especially $\mathrm{N}$, have different effects on the growth rate of plants ${ }^{[4]}$, dry matter distribution $^{[5]}$, nutrient uptake state ${ }^{[6]}$, soil physical and chemical properties $^{[7]}$, photosynthesis ${ }^{[8]}$, and carbon and nitrogen metabolism ${ }^{[2]}$. Among these, the carbon-nitrogen ratio $(\mathrm{C} / \mathrm{N})$ reflects the physiological status and growth vigor of the plant ${ }^{[9]}$. The $\mathrm{C} / \mathrm{N}$ is expressed in a variety of forms, including total carbon/total nitrogen ${ }^{[10]}$, (cellulose + lignin)/total nitrogen ${ }^{[11]}$, and soluble sugar/total nitrogen $(\mathrm{SS} / \mathrm{N})^{[12]}$. In this study, the $\mathrm{SS} / \mathrm{N}$ form was selected for study because tomato soluble sugar (SS) content is one of the most crucial internal quality factors, which can

\section{Received date: $2018-03-28 \quad$ Accepted date: $2020-01-16$}

Biographies: Jinyang Li, PhD, Associate Professor, research interest: biological information sensing, Email: by0817136@163.com; Lin Li, Assistant Researcher research interests: agricultural engineering, Email: lilin@ujs.edu.cn; Aichen Wang, Assistant researcher, research interests: agricultural engineering, Email: winterwac@163.com; Xinhua Wei, PhD, Professor, research interest: intelligent agricultural equipment, Email: wei_xh@126.com; Hanping Mao, PhD, Professor, research interest: intelligent agricultural equipment, maohp@ujs.edu.cn. *Corresponding author: Wenjing Zhu, $\mathrm{PhD}$, Assistant Researcher, research interests: agricultural information technology. Jiangsu University, Zhenjiang 212013, China. Tel: +86-511-88797338, Email: zwj0410@ foxmail.com. determine the fruit sweetness and flavor quality, in addition to being sensitive to nutritional status, and thus better reflects the plant growth status ${ }^{[13]}$. Plant physiology studies have already shown that the route of tomato fruit SS accumulation is mainly through two cytological pathways: apoplastic unloading and plastid pathway unloading ${ }^{[14]}$. Both methods require long-distance transport from the blade through the sieve tube, then unloading from the sieve element/companion cell complexes to the fruit ${ }^{[15]}$. Hence, the non-destructive testing of leaves is important for the diagnosis and management of $\mathrm{N}$ and $\mathrm{SS} / \mathrm{N}$ in tomato growth.

In recent years, numerous studies involving rapid estimation of plant leaf nutrient requirements have been carried out with hyperspectral imaging to replace time-consuming and costly traditional chemical analysis. Researchers attempted to apply hyperspectral imaging technique to diagnosis and estimate plant properties, such as nitrogen concentration in flue-cured tobacco leaves ${ }^{[3]}$, nitrogen deficiency effects on plant growth, leaf photosynthesis, and hyperspectral reflectance properties of sorghum $^{[16]}$, and chlorophyll concentration to display the nitrogen deficiency distribution map of cucumber leaves ${ }^{[17]}$. Analytical models were successfully developed to optimize remotely-sensed vegetation indices for retrieving leaf biochemical constituents ${ }^{[18,19]}$, to investigate changes at the leaf scale ${ }^{[20,21]}$ or canopy level ${ }^{[22,23]}$. In addition to detecting the major elements, an SS content model of oilseed rape leaves was built via a back-propagation neural network $^{[24]}$. Typical indices aimed at $\mathrm{N}$ estimation and chlorophyll evaluation were tested to assess leaf $\mathrm{C} / \mathrm{N}$ in winter wheat and spring barley ${ }^{[25]}$. Leaf $\mathrm{C} / \mathrm{N}$ ratios were estimated in three forest sites, with $R^{2}$ of the models being more than 0.8 with 
not more than 4 wavelengths being selected ${ }^{[26]}$. These studies show that using hyperspectral imaging technique to estimate plant $\mathrm{N}, \mathrm{SS}$, and $\mathrm{C} / \mathrm{N}$ status is feasible. However, these studies used sensors to collect leaf spectral information at a fixed angle, and focused on wavelength information or strength of the light source, such as reflectivity or reflection intensity. In reality, light intensity, wavelength, phase, and polarization contain large amounts of information, but polarization examination is rarely performed. The mechanisms of polarization reflection spectra can effectively improve the modeling, and be useful to the condition of isotropy diffuse reflection inversion. Since the 1970s, technologies for polarization reflection spectroscopy have rapidly developed, with applications in many research areas ${ }^{[27]}$. Polarized reflectance spectroscopy was applied in a variety of plants both at the single-leaf and canopy level, such as laurel (Laurus nobilis), mullein (Verbascum thapsus L. ${ }^{[28]}$, wheat (Triticum aestivum) ${ }^{[29]}$, chrysina gloriosa (Gloriosa superba L. $)^{[30]}$, arabidopsis (arabidopsis thaliana Linn. Heynh.) ${ }^{[31]}$ and citrus (Citrus reticulate $)^{[32]}$. Shibayama et al. ${ }^{[29]}$ discussed the volume, specular, and hot-spot scattering features of heading-stage wheat canopies. $\mathrm{Wu}$ et al. $^{[33]}$ found the five reflectance parameters of sedum spectabile boreau (Hylotelephium erythrostictum Miq. H. Ohba) were consistent for all spectral and spatial aspects by polarized hyperspectral imaging, and concluded that polarization-measured reflectance images can replace traditional remote sensing intensity reflectance images. In addition, Tan and $\mathrm{Khan}^{[34]}$ conducted polarimetric measurements of the backscattered light from lilac leaves and found that depolarization ratio was a good indicator of water stress.

Few investigations of tomato carbon and nitrogen metabolism by feature layer data fusion of polarized spectra-hyperspectral measurements have been reported, although tomato is cultivated in several Chinese provinces as a significant alimentary crop with large yield and planting area. Song et al. ${ }^{[35]}$ proposed that polarized reflectance features of leaves were mainly affected by viewing zenith angle, incident zenith angle, azimuth, and polarizer angle after they observed single maize (Zea mays L.) leaves. Liao et al. $^{[36]}$ investigated the effects of observation angle on the estimation of leaf area index of two types of summer maize, and established partial least square regression models; the results show that the simple ratio index model has obtained the highest estimation accuracy, with $\mathrm{R}^{2}$ of 0.47 and RMSE of 0.30 , by the combination of six observation angles. $\mathrm{Lü}^{[37]}$ measured the polarization of a corn canopy in different growth periods, at the nadir before heading, and found that polarized light accounts for up to $10 \%$ of the total reflection. Jay et al. ${ }^{[38]}$ evaluated the potential of nadir and off-nadir ground-based spectro-radiometric measurements to remotely sense five plant traits relevant for field phenotyping, namely, the leaf area index, leaf chlorophyll and

nitrogen contents, and canopy chlorophyll and nitrogen contents over fourteen sugar beet (Beta vulgaris L.) cultivars. They showed great potential to retrieve canopy nitrogen content, with RMSE $=10 \%$, while the estimation of leaf-level quantities was less accurate, with the best accuracy being RMSE $=17 \%$. Our research team assessed the feasibility of determining the nitrogen and potassium content of fresh, greenhouse-grown tomato leaves by using a self-developed polarization reflectance spectrum spectro-goniophotometer system, and found that it was more accurate to measure the nitrogen and potassium content with polarized models ${ }^{[39,40]}$.

In this study, we extract data from both the hyperspectral imaging system and polarized spectro-goniophotometer measurements to obtain complex information. We take into consideration both the characteristics of the continuous correlation of spectral data and the advantages of specific spatial angles, making the predictive model more robust. We evaluate the accuracy and feasibility of establishing predictive models of N, SS, and SS/N. Rapid detection combined with data fusion provides the theoretical basis and technical support for predicting the carbon and nitrogen metabolism parameters of crops.

\section{Materials and methods}

\subsection{Sample preparation}

Tomato (Lycopersicon esculentum Mill) seedlings with five true leaves from the Vegetable Research Institute, Academy of Agricultural Sciences, Liaoning Province, China, were transplanted individually into pots filled with perlite in a Venlo-type greenhouse at Jiangsu University in China $\left(32.11^{\circ} \mathrm{N}, 119.27^{\circ} \mathrm{E}\right)$. Plants were spaced in double rows at a density of $30 \mathrm{~cm}^{2}$ per plant. In contrast to conventional agriculture, the application and availability of nutrients can be monitored easily in greenhouse systems because each group of tomato roots had a fixed nutrient solution content delivered by a self-developed timed irrigation and collection system. The average temperature of the greenhouse was $23.26^{\circ} \mathrm{C}$, while the humidity was $55.18 \%$.

We induced five levels of $\mathrm{N}$ nutrition stress $(25 \%, 50 \%, 75 \%$, $100 \%$, and $150 \%$ ) to the tomato plants (Table 1). Yamazaki nutrient solution was used for irrigation; the nutrient solution was formulated to eliminate $\mathrm{NO}_{3}$ and $\mathrm{NH}_{4}$ without changing the concentrations of the other essential elements. The same trace element nutrient solution was supplied to all plants, and consisted of: EDTA-Na2Fe $20 \mathrm{mg} / \mathrm{L}, \mathrm{H} 3 \mathrm{BO} 32.86 \mathrm{mg} / \mathrm{L}, \mathrm{MnSO} 4.4 \mathrm{H} 2 \mathrm{O}$ $2.13 \mathrm{mg} / \mathrm{L}, \mathrm{ZnSO} 4.7 \mathrm{H} 2 \mathrm{O} 0.22 \mathrm{mg} / \mathrm{L}, \mathrm{CuSO} 4.5 \mathrm{H} 2 \mathrm{O} 0.08 \mathrm{mg} / \mathrm{L}$, and (NO4)6Mo7O24.4H2O $0.02 \mathrm{mg} / \mathrm{L}$. The solutions were supplied at a rate of $500 \mathrm{~mL} /$ plant.day for 1 month after transplanting, and $1000 \mathrm{~mL} / \mathrm{d} \cdot$ plant for an additional month until harvesting. Recommended pesticides (carbendazim, colloidal sulfur suspension agent) were used as needed to control greenhouse insects.

Table 1 Tomato nitrogen-stress macro-element solutions $\mathrm{mg} \cdot \mathrm{L}^{-1}$

\begin{tabular}{ccccccccc}
\hline $\mathrm{N}$ content & $\mathrm{Ca}\left(\mathrm{NO}_{3}\right)_{2}$ & $\mathrm{KNO}_{3}$ & $\left(\mathrm{NH}_{4}\right) \mathrm{H}_{2} \mathrm{PO}_{4}$ & $\mathrm{MgSO}_{4}$ & Complement $\mathrm{NaH}_{2} \mathrm{PO}_{4}$ & Complement $\left(\mathrm{NH}_{2}\right)_{2} \mathrm{CO}_{3}$ & $\mathrm{Complement} \mathrm{KCl}$ \\
\hline $25 \%$ & 89 & 101 & 19 & 246 & 60 & 0 & 0 & 224 \\
$50 \%$ & 177 & 202 & 39 & 246 & 40 & 0 & 149 \\
$75 \%$ & 266 & 303 & 58 & 246 & 20 & 0 & 75 \\
$100 \%$ & 354 & 404 & 77 & 246 & 0 & 4 & 0 \\
$150 \%$ & 354 & 404 & 77 & 246 & 0 & 0 \\
\hline
\end{tabular}

\subsection{Measurement of polarized spectra data}

The polarization reflectance spectra were collected using a device that was specifically designed for leaf bidirectional reflectance (BRDF) measurements. This device was developed by the Key Laboratory of Modern Agricultural Equipment and Technology ${ }^{[39]}$, Jiangsu University. The instrument was preheated 
for more than 1 hour to ensure that the instrument can work steadily. The operating mode of this device, and the BRDF measurement principle and angle range, were as described in our previous articles ${ }^{[39]}$. Light from a halogen light source was transmitted to an illumination manipulator through an optical fiber and produced unpolarized radiation in the VIS-NIR spectrum. The spectral range spans $300-1000 \mathrm{~nm}$; spectra were measured in $0.443 \mathrm{~nm}$ intervals, resulting in 1580 measurements. For each sample, the spectral collection was repeated at three points: the leaf tip, middle, and bottom, and the average curves were taken as the final scan result, to minimize the impact of random errors.

During the growing period, a total of five spectra were collected, corresponding to the five stages of tomato growth: seedling stage, flowering stage, initial fruiting stage, mid-fruiting stage, and picking stage. Developing a database of information on leaf analysis and fertility practices over time facilitates the diagnosis of problems as they occur. In total, 96 fresh leaves were collected for the determination of $\mathrm{N}$ and SS content. To avoid unwanted scattering, the laboratory must take dark measures to ensure a reflectance of less than $3 \%$ in the VIS-NIR spectrum. The temperature in the laboratory was maintained at $25^{\circ} \mathrm{C}$ and relative humidity at $40 \%$. A white reference panel (Spectralon, $\mathrm{BaSO} 4,99 \%$ reflectance) was used under the same conditions to convert the spectral reflectance before each test to minimize environmental influences, and the scan time was $0.2 \mathrm{~s}$. The raw polarization spectra of $\mathrm{N}$ stress samples are presented in Figure 1.

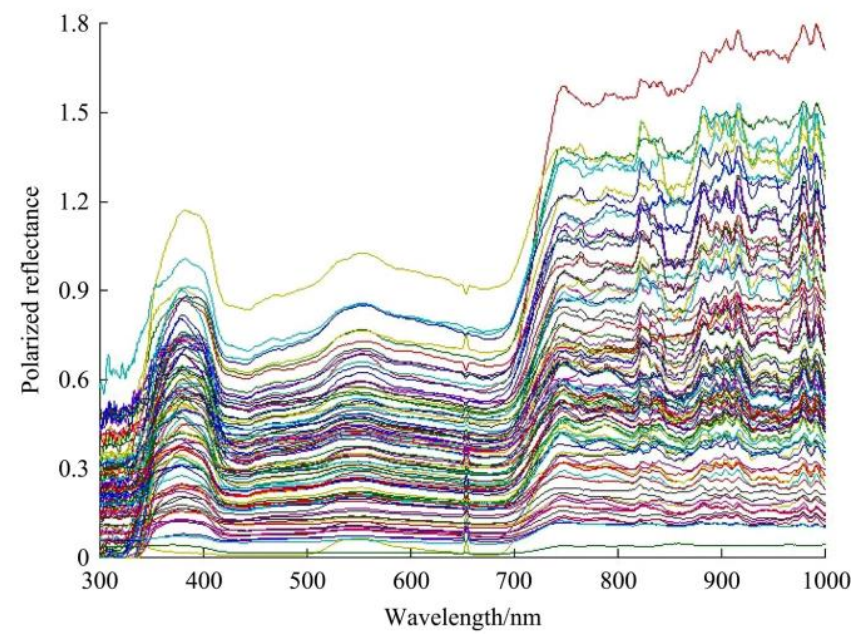

Figure $1 \quad 0^{\circ}$ Polarization spectra of $96 \mathrm{~N}$ nutrition stress samples

\subsection{Measurement of hyperspectral data}

Hyperspectral data of tomato leaves were collected by a hyperspectral imaging system. The system was mainly composed of a hyperspectral camera (V10E-QE, Spectral Imaging Ltd., Finland), a $150 \mathrm{~W}$ halogen lamp DC tunable light source (2900-ER +9596-E, Illumination Technologies, USA), two-fiber line light source (PIN 9145+9530, Illumination Technologies, USA), moving stage (MTS120, Beijing Optical Instrument Factory, China), controller (SC100, Beijing Optical Instrument Factory, China), and computer components. Before the hyperspectral image data acquisition, the exposure time of the visible-near infrared camera was determined in advance to ensure that the image was clear and the speed of the displacement table was determined to avoid the distortion of the image size and spatial resolution. The exposure time was determined after analysis and comparison to be $20 \mathrm{~ms}$, and the displacement speed of the displacement table was $1.25 \mathrm{~mm} / \mathrm{s}$. The spectral range was $300-1000 \mathrm{~nm}$ while the spectral resolution was $2.8 \mathrm{~nm}$. The sampling interval was $1.2 \mathrm{~nm}$; thus, a single acquisition can obtain 512 independent hyperspectral images. The black and white fields must be calibrated to set the reflectivity range before the data acquisition, and then the second-order Butterworth filter is used for digital filtering to remove noise interference.

\subsection{Chemical measurement}

The chemical content determinations were performed in parallel with the spectral test. The leaf samples were picked between 9:00 and 12:00 am Beijing local time into numbered self-styled bags, then sorted into the professional plant preservation box, immediately transported to the laboratory, and then polarized reflection spectrum measurement experiments and hyperspectral image acquisition were performed. After collection, the leaves were placed in an oven and dried at $80^{\circ} \mathrm{C}$ until a constant weight was achieved. The total nitrogen content of the samples was determined by the Kjeldahl method. Concentrated sulfuric acid $(5 \mathrm{~mL})$ was added to each sample, and samples were heated to $380^{\circ} \mathrm{C}$ for $4 \mathrm{~h}$ according to the Kjeldahl protocol. The $\mathrm{N}$ content of the concentrates was then determined using a continuous flow AutoAnalyzer 3 (SEAL Analytical Instruments, USA). All chemical reagents used in the analyses were of analytical grade; results were expressed on a fresh-weight basis $(\mathrm{mg} / \mathrm{g})$.

The anthrone colorimetric method was used to determine the SS content in the leaves. $0.1 \mathrm{~g}$ of fresh plant leaves were placed in test tubes, $5 \mathrm{ml}$ of distilled water was added, a plastic film used for sealing, and a boiling water bath extraction for $30 \mathrm{~min}$ (repeated extraction 2 times) was performed. The extract was filtered into a $25 \mathrm{ml}$ volumetric flask. $1 \mathrm{ml}$ of the extract was transferred from the volumetric flask to the inside of the tube, $0.5 \mathrm{ml}$ of anthrone ethyl acetate and $5 \mathrm{ml}$ of concentrated sulfuric acid were added in sequence, and the mixed liquid was sufficiently shaken and placed in a boiling water bath for $30 \mathrm{~min}$. After cooling to room temperature for $10 \mathrm{~min}$, the absorbance was measured at a wavelength of $630 \mathrm{~nm}$. The SS content was calculated based on the standard curve that has been drawn using glucose as a standard sample.

The SS/N is the sugar and nitrogen ratio of the mass fraction and is a dimensionless index obtained by dividing the mass fraction of soluble sugar $S S$ by the mass fraction of total nitrogen $N$, and multiplying by the conversion factor $F$ according to

$$
S S / N=\frac{S S}{N} \times F \times 100 \%
$$

\subsection{Software and statistical analysis}

All data processing and analysis were performed using MATLAB Version 2010a (Mathworks, USA) for Windows 7. BRDF analyzer software (Isuzu Optics, Taiwan) was used for the acquisition and analysis of raw BRDF spectral data. ENVIV4.5 (Research System, Inc, USA) was used for the acquisition and analysis of raw hyperspectral data. The precisions of the model and validated model were evaluated with the root mean square error of cross-validation (RMSECV), the root mean square error of prediction (RMSEP), and the correlation coefficient (R). Higher $\mathrm{R}$, lower RMSECV, and lower RMSEP indicate the higher precision and accuracy of a model.

\section{Results and discussion}

\subsection{Extraction of optimum polarization degree features}

3.1.1 Plant physiological analysis of polarization reflections based on scanning electron microscopy

In order to show that there is a link between the nutritional 
stress and the microstructure of the tomato leaves, the surface microstructures of the $\mathrm{N}$-deficient and $\mathrm{N}$-excess leaves were analyzed by scanning electron microscopy compared to the normal leaves. Figures 2a-2c show the surface microstructures of normal, $\mathrm{N}$-deficient, and $\mathrm{N}$-excess tomato leaves at $\times 200$ magnification, respectively.

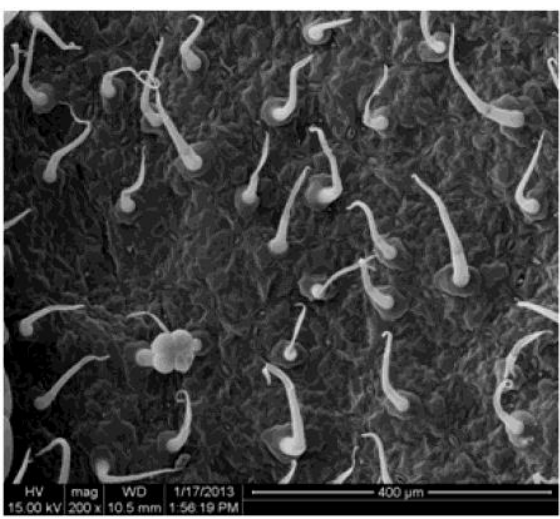

a. Control group, $\times 200$

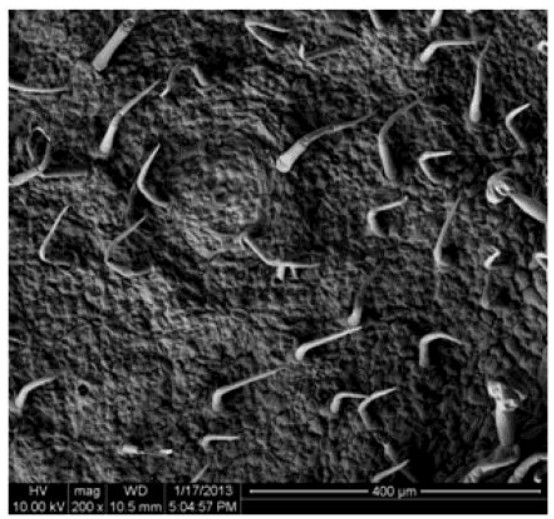

b. $\mathrm{N}$ deficiency, $\times 200$

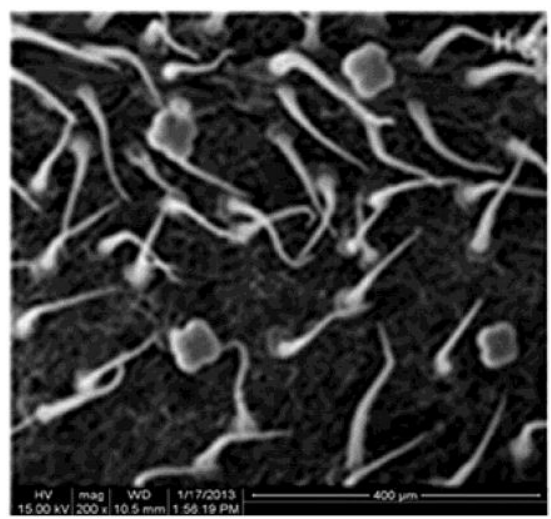

c. $\mathrm{N}$ excess, $\times 200$

Figure 2 Scanning electron micrographs of N-stressed leaves

In Figure 2a, the pores can be seen clearly, the blade surface is mainly distributed with tapered villi and a small number of mushroom-shaped villi, as well as protruding vascular bundles. Defensive cells were half-moon shaped, and the cilia stretch well. Although the epidermal cells are irregular in shape, they are tightly fitted with each other. Due to the good growth of the chloroplast, the corpus cavernous bodies, and the fence tissue, the blade surface has a uniform water-like structure, and the mesophyll morphology is plump. Most of the cilia lodged, and the stomas have closed due to lack of $N$ (Figure $2 b$ ). Significantly more folds and cracks on the surface of the leaf compare to Figure 2a, the epidermal cells are dry and contraction because of undernourishment. The leaf looks more withered and scorched. Figure $2 \mathrm{c}$ shows that the blade tissue is loose and the folds are obvious, due to the application of excess $\mathrm{N}$, resulting in excessive development of the stratum corneum and greater thickness than normal leaves. The incident light is reflected by the surface of the object, resulting in polarization degree features, which are mainly controlled by two factors: the refractive index of the object, and the incident angle. The change of polarization parameters caused by the severity of nutrient stress of tomato leaves was investigated, and the polarization parameters were judged and selected according to the following rule: Orthogonal experiments and range analyses were performed to verify the optimum angle combination from the polarized reflectance parameters. Optimum angle combination experiments were then conducted to fine-tune the optimal parameters, which resulted in the following conditions: incident zenith angle, $60^{\circ}$; viewing zenith angle, $45^{\circ}$; light source polarizer, $0^{\circ}$; detector polarizer, $45^{\circ}$; and azimuth, $180^{\circ}$. The specific selection method was as described in our previous articles ${ }^{[39,40]}$.

3.1.2 Extraction of polarization degree features

Since there is significant noise in the hyperspectral spectrum below $440 \mathrm{~nm}$ and above $950 \mathrm{~nm}$, polarization data from 440-950 nm were selected for analysis. The average polarization spectrum of each tomato sample in the $0^{\circ}, 90^{\circ},+45^{\circ},-45^{\circ}$ direction was extracted. Hence, the polarization degree of each sample was calculated at different wavelengths according to the Stokes formula $^{[41]}$ :

$$
S=\left[\begin{array}{l}
I \\
Q \\
U \\
V
\end{array}\right]=\left[\begin{array}{l}
I_{0^{\circ}}+I_{90^{\circ}} \\
I_{90^{\circ}}-I_{0^{\circ}} \\
I_{+45^{\circ}}-I_{-45^{\circ}} \\
I_{r}+I_{l}
\end{array}\right]
$$

Figure 3 shows the polarization degree for different nutritional stresses, in which it can be seen that the polarization degree increases with the increase of nitrogen supply level. The polarization degree is maintained at a relatively high level between $350-680 \mathrm{~nm}$, then the curve suddenly drops at $680-720 \mathrm{~nm}$; the near-infrared region shows a lower degree of polarization and the curve tends to become flat beyond $720 \mathrm{~nm}$. The mean values of polarization in the five growth stages were correlated with the chemical reference value of $\mathrm{N}$, as shown in Figure 4 , where a distinguishing line of significance level $\alpha=0.01$ is shown to estimate the probability that the overall parameter may fall within a certain interval. The smaller the value of $\alpha$, the higher the confidence value or confidence level, and interval estimates are more reliable.

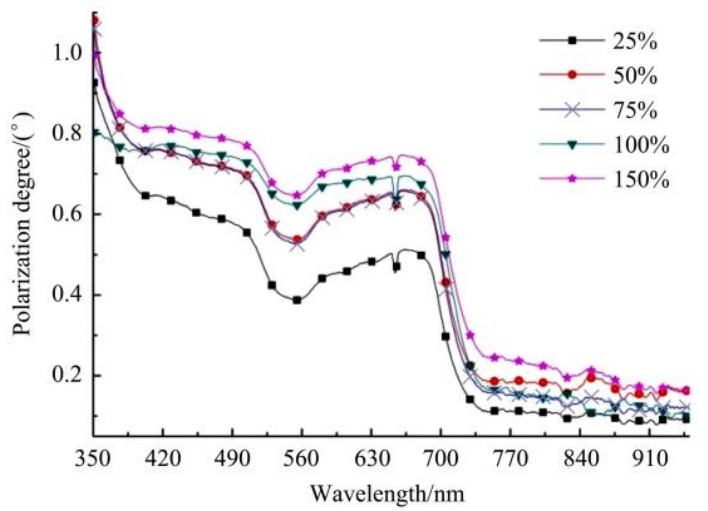

Figure 3 Polarization degree for different nutritional stresses

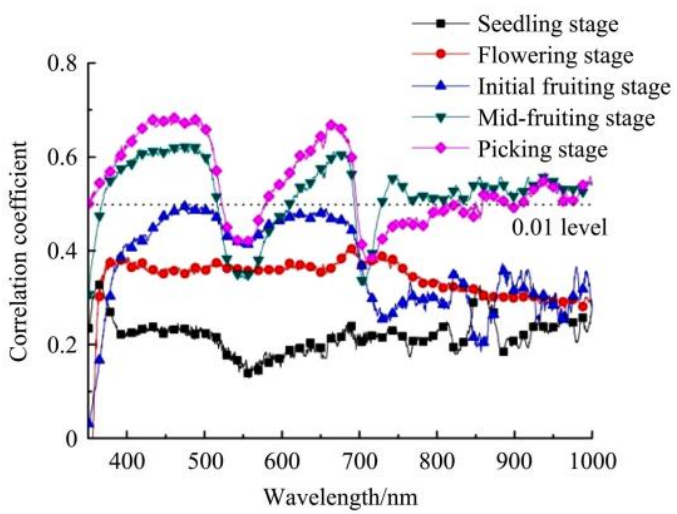

Figure 4 Correlation analysis between polarization degree and $\mathrm{N}$-content of tomato leaves 
The specific steps of wavelength extraction are as follows: firstly, the sensitive band obtained by correlation analysis was divided into several wavelength groups, with each group containing 10 wavelengths; secondly, each group was sorted to form a subset of $U i$ according to the value of the relevant coefficient; thirdly, the band with the largest correlation coefficient was set to $U s$; finally, the bands in $U i$ were compared with the $U s$ individually, and if the correlation coefficients with all the bands in $U s$ were less than 0.8 , they were selected. The final results for sensitive wavelengths obtained by the method were $380.49 \mathrm{~nm}, 655.41 \mathrm{~nm}, 744.48 \mathrm{~nm}$, and $850.58 \mathrm{~nm}$.

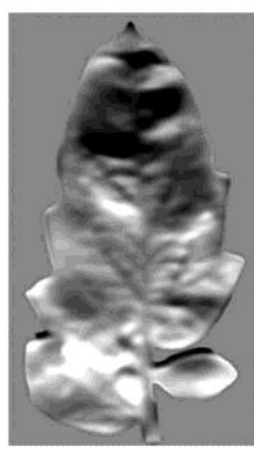

PC1

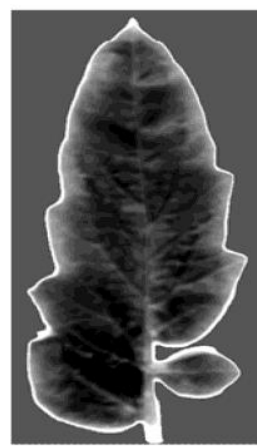

PC2

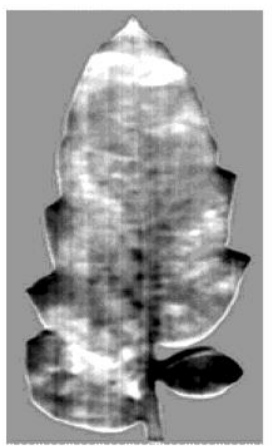

$\mathrm{PC} 3$

\subsection{Feature extraction of hyperspectral data}

3.2.1 Extraction of texture features under sensitive wavelengths

Principal component analysis (PCA) was used to reduce the spectral dimension after a $5 \times 5$ window median filter was applied, and image segmentation at $700 \mathrm{~nm}$, because the difference between the background and the leaf at $700 \mathrm{~nm}$ was the most pronounced. Figure 5 shows images of the first five principal components (PCs) of tomato leaves. The total accumulative contribution rate of variance from the top five PCs (PC1, PC2, PC3, PC4, and PC5) was $99.00 \%$, which means that the top five scores for the 120 samples can explain $99.00 \%$ of the raw spectral information from all samples.

Figure 5 PC images of the first five PCs of the mildly-stressed $(N=75 \%)$ tomato leaf

The weight coefficients of the first five principal components were calculated and plotted using PCA analysis. The weight coefficients can represent the importance of an indicator item in the overall system. Here, by analyzing the weight coefficient of the first five principal components of the $\mathrm{N}$-stressed leaves, the maximum absolute value of the weight coefficient can be shown, which indicates that these wavelengths contribute most to the reaction N-stress. Figure 6 shows the weight coefficients of the $\mathrm{N}$-content of the first five PCs after PCA; the final results for sensitive wavelengths were $464.91 \mathrm{~nm}, 566.29 \mathrm{~nm}, 696.28 \mathrm{~nm}$, and $724.66 \mathrm{~nm}$.

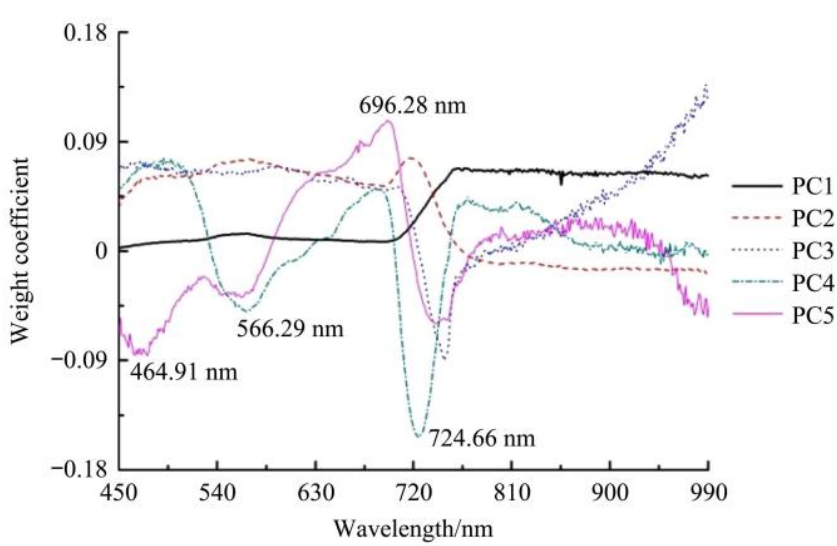

Figure 6 Weight coefficient of the N-content of the first five PCs after PCA

Eight texture features according to the gray level co-occurrence matrices (GLCM) were extracted based on the above four sensitive wavelengths: Mean (MEA), Variance (VAR), Homogeneity (HOM), Entropy (ENT), Dissimilarity (DIS), Angular Second Moment (ASM), Contrast (CON), and Correlation (COR). These texture features are the most representative and persuasive in the study of plant nutrient stress ${ }^{[42]}$.

$$
M E A=\sum_{i=0}^{L-1} \sum_{j=0}^{L-1}(i, j) p(i, j)
$$

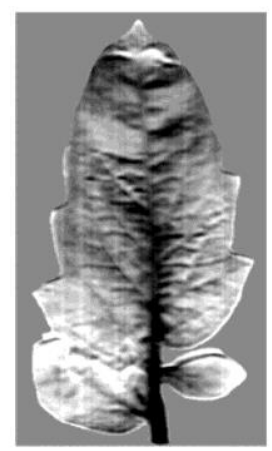

PC4

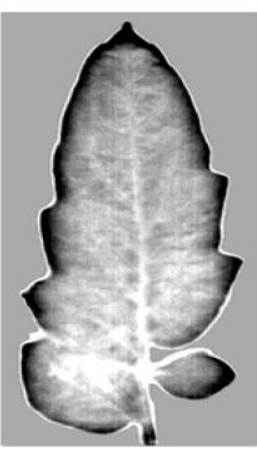

PC5

$$
V A R=\sum_{i=0}^{L-1} \sum_{j=0}^{L-1}\left(i-u_{x}\right)\left(j-u_{y}\right) p(i, j)
$$

where,

$$
\begin{gathered}
u_{x}=\sum_{i=0}^{L-1} i \sum_{j=0}^{L-1} p(i, j) ; u_{y}=\sum_{i=0}^{L-1} j \sum_{j=0}^{L-1} p(i, j) \\
H O M=\sum_{i=0}^{L-1} \sum_{j=0}^{L-1} \frac{p(i, j)}{1+|i-j|} \\
E N T=-\sum_{i=0}^{L-1} \sum_{j=0}^{L-1} p(i, j) \ln p(i, j) \\
D I S=\sum_{i=0}^{L-1} \sum_{j=0}^{L-1} \frac{p(i, j)}{1+|i-j|^{2}} \\
A S M=\sum_{i=0}^{L-1} \sum_{j=0}^{L-1}(p(i, j))^{2} \\
\text { CON }=\sum_{i=0}^{L-1} \sum_{j=0}^{L-1}(i-j)^{2} p(i, j) \\
\text { COR }=\frac{\left\{\sum_{i=0}^{L-1} \sum_{j=0}^{L-1} i j p(i, j)\right\}-u_{x} u_{y}}{\sigma_{x} \sigma_{y}}
\end{gathered}
$$

where, $u_{x}=\sum_{i=0}^{L-1} i \sum_{j=0}^{L-1} p(i, j) ; \quad u_{y}=\sum_{i=0}^{L-1} j \sum_{j=0}^{L-1} p(i, j) ;$

$$
\sigma_{x}=\left(\sum_{i=0}^{L-1}\left(i-u_{x}\right)^{2} \sum_{j=0}^{L-1} p(i, j) ; \quad \sigma_{y}=\left(\sum_{i=0}^{L-1}\left(j-u_{y}\right)^{2} \sum_{j=0}^{L-1} p(i, j)\right.\right.
$$

Table 2 Correlation coefficients between texture features and $\mathrm{N}$-content at the sensitive wavelengths

\begin{tabular}{ccccccccc}
\hline $\begin{array}{c}\text { Sensitive } \\
\text { wavelength }\end{array}$ & MEA & VAR & HOM & CON & DIS & ENT & ASM & COR \\
\hline $464.91 \mathrm{~nm}$ & -0.4558 & -0.2483 & 0.4703 & -0.5850 & -0.5038 & -0.5795 & 0.6892 & 0.6798 \\
$566.29 \mathrm{~nm}$ & -0.3941 & 0.4420 & -0.6772 & 0.6711 & 0.4235 & 0.1034 & -0.6028 & 0.3546 \\
$696.28 \mathrm{~nm}$ & 0.0971 & 0.6867 & -0.2792 & 0.4900 & 0.6578 & 0.1184 & -0.1010 & 0.2316 \\
$724.66 \mathrm{~nm}$ & 0.4593 & 0.5553 & -0.5814 & 0.3862 & 0.4290 & 0.5804 & -0.5854 & 0.6840 \\
\hline
\end{tabular}


Correlation analysis of the eight features and the chemical reference value of $\mathrm{N}$ content were implemented, as shown in Table 2. The texture features with the highest correlation values were chosen for final modelling: VAR ${ }_{696.28}, H M_{566.29}, C_{56} N_{566.29}$, $D I S_{696.28}, A S M_{566.29}$, and $C O R_{724.66}$. The correlation values of MEA and ENT under four sensitive wavelengths are all below \pm 0.6 , non-adoption. Texture feature is often used as a feature for image classification and information extraction when the spectral features of ground objects are similar. It is formed by the repeated occurrence of gray distribution in the spatial position. Therefore, there is a certain gray relationship between two pixels separated by a certain distance in the image space, that is, the spatial correlation characteristics of gray in the image. Texture features are not based on pixel features it needs to be calculated in the area containing multiple pixel points. In pattern matching, this kind of regional feature has great advantages and cannot be matched successfully because of local deviation. The correlation values of MEA and ENT under four sensitive wavelengths are all below \pm 0.6 which illustrate that these two methods have fewer advantages in pattern matching and lower matching success rate.

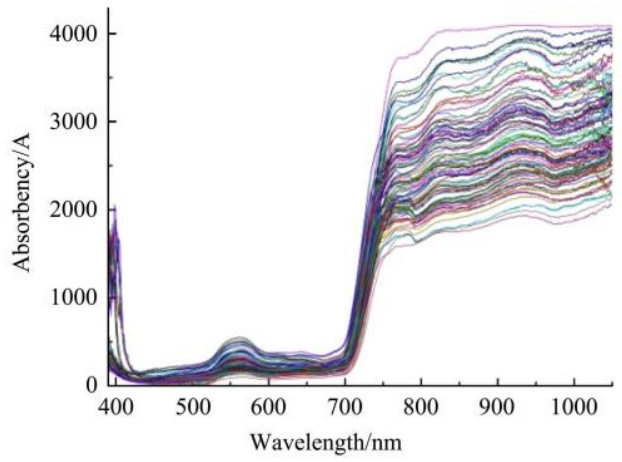

a.
3.2.2 Optimal reflection spectrum features selected by iPLS-GA

Figure $7 \mathrm{a}$ presents the raw spectra profile of all the samples. Raw spectra contained background information and noise in addition to the sample information. Spectral pretreatment is an effective mean to overcome spectroscopic instability. Traditional methods of spectral pre-processing include smoothing, derivative, standard normal variate transformation (SNV), multiplicative scatter correction, Fourier transform, and $\mathrm{min} / \mathrm{max}$ normalization ${ }^{[43]}$. The best performance was shown by SNV, which was thus selected for this study. SNV transformation was performed for each spectrum, individually, by subtracting the mean of the spectrum and scaling with the standard deviation of the spectrum, as illustrated by:

$$
x_{i, \mathrm{SNV}}=\frac{x_{i}-\bar{x}}{\sqrt{\sum_{i=1}^{n}\left(x_{i}-\bar{x}\right)^{2} /(n-1)}}
$$

where, $x i_{, \mathrm{SNV}}$ is the SNV transformed spectral value for the ith variable; $x_{i}$ is the ith variable in the raw spectrum, and $\bar{x}$ is the mean of the raw spectrum ${ }^{[44]}$. The spectra after SNV preprocessing are presented in Figure $7 \mathrm{~b}$.

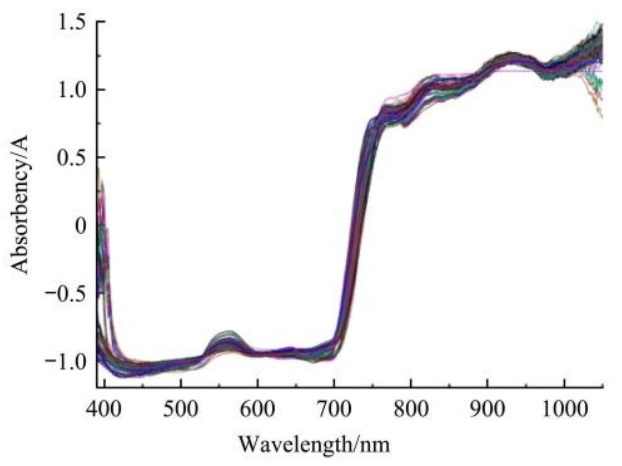

b.

Figure 7 Raw spectra (a) and SNV preprocessed spectra (b) of tomato leaf samples

The spectral preprocessing method cannot eliminate all the invalid information such as instrument noise and sample background noise contained in the original spectrum; therefore, it is necessary to optimize the independent variables and extract the effective information from the redundant data. Hence, the reduced model predictive ability and robustness will be enhanced. In this study, we used an interval partial least squares-genetic algorithm (iPLS-GA) to optimize the variables. The most prominent advantage of the iPLS approach is to find out the most relevant intervals for the quality of the test ${ }^{[45]}$. The GA simulates the natural genetic mechanism and natural selection of the biological world and is more suitable for solving complex, nonlinear optimization problems ${ }^{[46]}$. The sensitive wavelengths of the $\mathrm{N}$ were found to be $741.48 \mathrm{~nm}, 755.74 \mathrm{~nm}, 767.44 \mathrm{~nm}$, and $784.37 \mathrm{~nm}$ using iPLS-GA.

\subsection{Model calibrations}

All 96 samples for each nutrient element were randomly divided into two subsets: a calibration set, used to build the model, and a prediction set, used to test the robustness of the model. To avoid bias in the division of the subsets, the division was performed as follows: all samples were sorted according to their respective $y$-value (the measured reference value of $\mathrm{N}$ and SS content). Two abnormal samples were removed according to the Mahalanobis distance. To reach a 2/1 division of calibration/prediction spectra, one spectrum from every three samples was added to the prediction set, so that the final calibration set contained 80 spectra and the remaining 40 spectra constituted the prediction set. The range of $y$-values in the calibration set covered the range in the prediction set; therefore, the distribution of the samples was appropriate (Table 3).

Table 3 Reference measurement results for samples

\begin{tabular}{ccccccc}
\hline \multirow{2}{*}{ Nutrients } & \multirow{2}{*}{ Units } & Set & S.N. & Range & Mean & S.D. $^{\text {b }}$ \\
\hline \multirow{2}{*}{$\mathrm{N}$} & $\%$ & Calibration set & 70 & $3.40-8.08$ & 5.37 & 2.35 \\
& $\%$ & Prediction set & 35 & $3.85-7.92$ & 5.29 & 2.21 \\
\hline \multirow{2}{*}{ SS } & $\%$ & Calibration set & 70 & $0.81-7.59$ & 4.02 & 2.69 \\
& $\%$ & Prediction set & 35 & $0.92-7.08$ & 3.93 & 2.47 \\
\hline
\end{tabular}

Note: ${ }^{\text {a }}$ S.N.: sample number. $\quad{ }^{\text {b }}$ S.D.: standard deviation.

A total of 14 feature variables were used for modeling, including 4 polarization degree features at $380.49 \mathrm{~nm}, 655.41 \mathrm{~nm}$, $744.48 \mathrm{~nm}$, and $850.58 \mathrm{~nm} ; 6$ texture features $\left(V A R_{696.28}, H_{0 M} M_{566.29}\right.$ $C O N_{566.29}, D I S_{696.28}, A S M_{566.29}$, and $C O R_{724.66}$ ), and 4 reflection spectrum features at $741.48 \mathrm{~nm}, 755.74 \mathrm{~nm}, 767.44 \mathrm{~nm}$, and $784.37 \mathrm{~nm}$.

Linear models (Multiple Linear Regression (MLR) ${ }^{[47]}$ and Partial Least Squares (PLS)), and nonlinear models (Support Vector Machine (SVM) ${ }^{[48]}$ and Back propagation Artificial Neural Network (BPANN) ${ }^{[49]}$ ) were both applied comparatively in model calibration. From the results for predicting N, the best data fusion model was achieved by BPANN with Rc $=0.9845$, RMSEC $=$ 0.1031 , and $\mathrm{Rp}=0.9400, \mathrm{RMSEP}=0.1995$. Figure 8a illustrates the predicted and measured values of $\mathrm{N}$ for the calibration and prediction sets using BPANN. Among the SS results, the best data fusion model for prediction was also achieved by BAPNN with $\mathrm{Rc}=0.9845$, RMSEC $=0.2893$, and $\mathrm{Rp}=0.9315$, and $\mathrm{RMSEP}=0.5559$. Figure $8 \mathrm{~b}$ illustrates the predicted and 
measured values of SS for the calibration and prediction sets using BPANN. After normalization and optimization, the best data fusion model for SS/N prediction was achieved by SVM with Rc = 0.9679 , RMSEC $=0.0077$, and $\mathrm{Rp}=0.9466$, RMSEP $=0.0259$, and corresponding to $\mathrm{C}=16$ and $\mathrm{g}=0.5$. Figure $8 \mathrm{c}$ shows the predicted and measured values of $\mathrm{SS} / \mathrm{N}$ for the calibration and prediction sets using SVM. As investigated from the results, the six models performed well in the prediction of $\mathrm{N}, \mathrm{SS}$, and SS/N while using the data fusion as the model input.

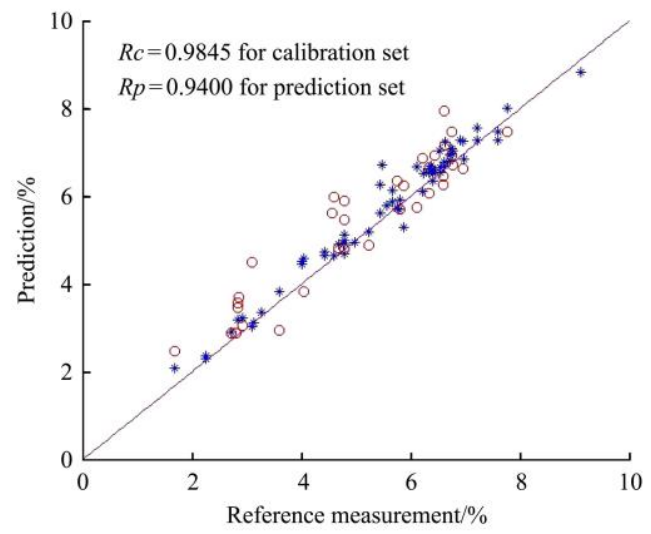

a.

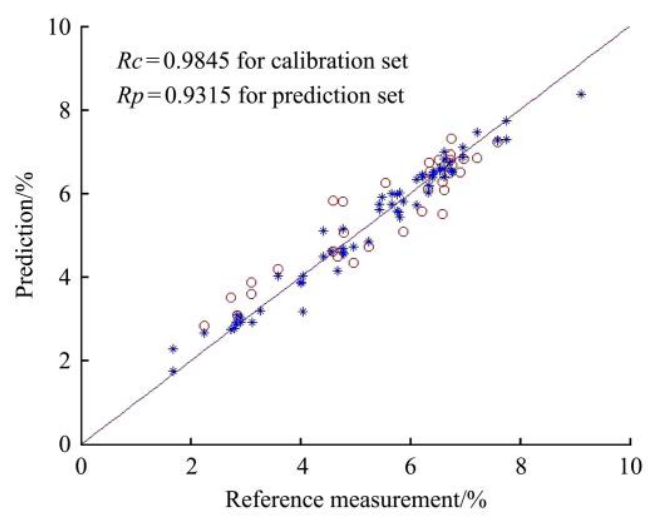

$\mathrm{b}$

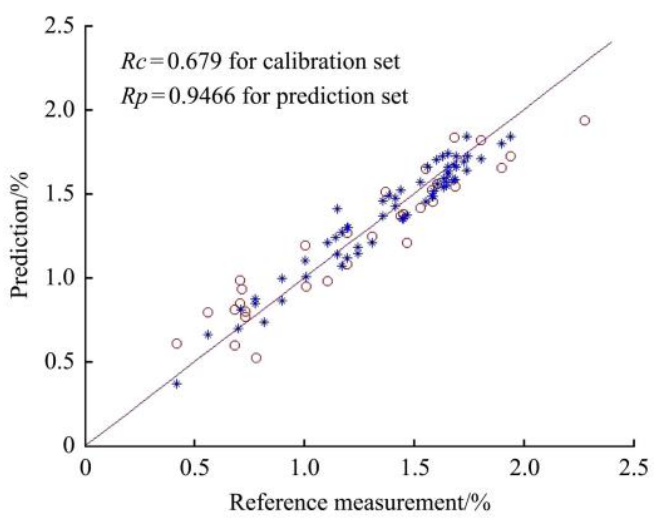

c.

Figure 8 (a) Reference determination versus of calibration (*) and prediction (o) set data of BP-ANN model of N.

(b) Reference determination versus of calibration $(*)$ and prediction (o) set data of the BP-ANN model of SS.

(c) Reference determination versus of calibration (*) and prediction (o) set data of the SVM model of SS/N

\subsection{Discussion}

The combination of polarization reflectance spectra and hyperspectral data analysis was systematically studied in this work and achieved a good performance in quantitatively measuring nutrition in tomato leaves, compared with the individual results of the two systems. The detailed discussion of the results can be summarized as follows.

Regarding the effect of different nitrogen levels on SS accumulation, the five growth cycle stages of the tomato plant correspond to different nitrogen gradient levels, and many other elements and trace elements are normally supplied. The distribution of SS content in each growth cycle was analyzed and some conclusions can be drawn. Firstly, with the continuous growth of tomato plants, the accumulation of SS showed a steady growth trend. Studies have shown that tomato SS gradually increases in the mid-term and harvest period, especially glucose and fructose, and reaches the highest level at maturity. This may be related to the change of sucrase activity, because it is reported that the sucrase activity is gradually increased with the development of the fruit, and reaches a maximum, resulting in the strongest hydrolysis of sugar ${ }^{[50]}$. Secondly, in one of the five growth cycles, the SS content showed a gradual decrease with the increase of nitrogen application rate. In short, the amount of nitrogen applied was negatively correlated with the content of SS in tomato leaves. This is not consistent with the report of $\mathrm{Li}$ Yuanxin, whose study showed that nitrogen fertilizer can increase the tomato SS content ${ }^{[51]}$. This is because the object of Yuanxin's detection was tomato fruit, while the object of this study is the leaf. After the fruit is developed, the fruit is a strong metabolic pool, and the leaves need to transport more SS to the fruit. This result is consistent with Wang Li's study, which pointed out that the content of SS in the leaves decreased rapidly 25 days after the flowering time and early fruiting period ${ }^{[50]}$. Moreover, in the early stage of nitrogen deficiency, plant leaf color is not very different from the naked eye, making it difficult to distinguish whether the virus is infected only with color characteristics ${ }^{[52]}$. Texture parameters, such as ENT, CON, and HOM, may change according to leaf microstructure structure and color shading in the canopy ${ }^{[42]}$. However, the image and reflectance spectra cannot be fully captured at the early stage when the leaf changes are not obvious. The degree of polarization calculated by the scanning polarization reflected light contains information related to the surface and inner layer of the leaf ${ }^{[28,53]}$. In this study, polarization detection is regarded as an effective supplement, and it is believed that polarization measurement can obtain some information that cannot be obtained by traditional intensity measurement, and thus can effectively improve the model accuracy.

The limitations of computer vision technique to evaluate nutrition deficiency in tomato leaves lead to the idea of introducing a fusion technique by combining data from two systems, which considers texture, morphology as well as color features.

Finally, in order to highlight the efficiency of the data fusion methodology proposed in this study, models calibrated with separate data sources were compared. Table 4 shows the results from the separate features and models of data fusion for N, SS, and $\mathrm{SS} / \mathrm{N}$. In this table, $M_{p}, M_{t}$, and $M_{r}$ represent models built by polarization degree features, texture features, and reflection spectrum features, respectively. $M_{p}+M_{t}+M_{r}$ represents the data fusion model. The best model from each separate feature $\left(M_{p}, M_{t}\right.$, and $M_{r}$ ) is also shown in Table 4, they conducted all the possibilities and found the optimum combination. It can be observed that the data fusion models achieved better performance than the separate feature models, regardless of the linear model (MLR, PLS) or the nonlinear model (SVM and BPANN). In addition, the SS/N models obtained sharply increased predictive 
accuracy. This proved that the information extracted from the polarized spectrum, reflection spectrum, and texture data, were mutually complementary, and the models could provide better results. In addition, among the models based on data fusion, the results did not have significant differences take N, SS, or SS/N into consideration separately.

Table 4 Evaluation of the data fusion models

\begin{tabular}{|c|c|c|c|c|c|c|c|c|c|c|c|c|c|}
\hline \multirow{2}{*}{ Models } & \multirow{2}{*}{ Methods } & \multicolumn{4}{|c|}{$\mathrm{N}$} & \multicolumn{4}{|c|}{ SS } & \multicolumn{4}{|c|}{$\mathrm{SS} / \mathrm{N}$} \\
\hline & & $R_{C}$ & RMSEC & $R_{P}$ & RMSEP & $R_{C}$ & RMSEC & $R_{P}$ & RMSEP & $R_{C}$ & RMSEC & $R_{P}$ & RMSEP \\
\hline$M_{p}$ & PLS & 0.9283 & 0.5426 & 0.9087 & 0.7465 & 0.6922 & 1.1527 & 0.6426 & 1.2392 & 0.7402 & 1.2552 & 0.7046 & 1.3085 \\
\hline$M_{t}$ & MLR & 0.9633 & 0.3832 & 0.9183 & 0.4525 & 0.9321 & 0.3216 & 0.7937 & 0.7965 & 0.8797 & 0.8202 & 0.8207 & 1.4568 \\
\hline$M_{r}$ & MLR & 0.9044 & 0.6524 & 0.8678 & 0.9817 & 0.8848 & 1.1598 & 0.7510 & 1.8761 & 0.7128 & 1.5964 & 0.5584 & 1.5507 \\
\hline$M_{p}+M_{t}+M_{r}$ & MLR & 0.9624 & 0.5006 & 0.9373 & 0.5448 & 0.9483 & 0.5205 & 0.9142 & 0.6139 & 0.9378 & 0.1211 & 0.9315 & 0.1734 \\
\hline$M_{p}+M_{t}+M_{r}$ & PLS & 0.9434 & 0.5161 & 0.9310 & 0.5921 & 0.9074 & 0.6838 & 0.9075 & 0.6544 & 0.9351 & 0.1236 & 0.9307 & 0.1738 \\
\hline$M_{p}+M_{t}+M_{r}$ & SVR-GS & 0.9562 & 0.1166 & 0.9291 & 0.2217 & 0.9520 & 0.0047 & 0.9147 & 0.0068 & 0.9679 & 0.0077 & 0.9466 & 0.0259 \\
\hline$M_{p}+M_{t}+M_{r}$ & SVR-PSO & 0.9521 & 0.1268 & 0.9289 & 0.2215 & 0.9389 & 0.0061 & 0.9136 & 0.0068 & 0.9663 & 0.0081 & 0.9422 & 0.0285 \\
\hline$M_{p}+M_{t}+M_{r}$ & BP-ANN & 0.9845 & 0.1031 & 0.9400 & 0.1995 & 0.9845 & 0.2893 & 0.9315 & 0.5559 & 0.9859 & 0.0585 & 0.9254 & 0.1794 \\
\hline
\end{tabular}

This combination of two sets of data could produce different responses and may provide further information. Thus, the data fusion technique of different modalities ${ }^{[54]}$ could provide better information compared to a single modal system. If the eigenvector is too large, the running time and effort of the fusion system will be affected. Nowadays, there are many information resources and the requirement of information processing ability is increasing. Data fusion technology can help people deal with complex information processing and judgment, and has high precision, practicability and feasibility.

\section{Conclusions}

This paper proposed a novel data fusion methodology of feature variables for an advanced instrumental measurement of plant physiological indexes. $\mathrm{N}, \mathrm{SS}$, and $\mathrm{SS} / \mathrm{N}$ in tomato leaves were used to verify the feasibility of this methodology. The proposed data fusion methodology showed obvious superiority in both predictability and stability of models in contrast to traditional methodologies. It can be concluded that this method is not as limited by traditional computer vision and spectroscopy technology, especially regarding the poor accuracy of the type of nutrition determination. It provides a theoretical basis for the establishment of a more precise automatic greenhouse system and promises to be significant in the development of precision agriculture technology.

\section{Acknowledgements}

The authors are grateful to the financial support by China national key research and development plan (2017YFD0700504), China Postdoctoral Science Foundation (2016M601743), Senior talent research start-up fund of Jiangsu University (14JDG151), Natural Science Foundation of China Youth Fund(61901194), Natural Science Youth Foundation of Jiangsu Province (BK20180863, BK20180861) and Priority Academic Program Development of Jiangsu Higher Education Institutions (PAPD-2018-87)

\section{[References]}

[1] Lequeue G, Draye X, Baeten V. Determination by near infrared microscopy of the nitrogen and carbon content of tomato (Solanum lycopersicum L.) leaf powder. Sci Rep, 2016; 6: 33183.

[2] Schlüter U, Mascher M, Colmsee C, Scholz U, Bräutigam A, Fahnenstich $\mathrm{H}$, et al. Maize source leaf adaptation to nitrogen deficiency affects not only nitrogen and carbon metabolism but also control of phosphate homeostasis. Plant Physiology, 2012; 160(3): 1384-1406.

[3] Jia F F, Liu G S, Liu D S, Zhang Y Y, Fan W G, Xing X X. Comparison of different methods for estimating nitrogen concentration in flue-cured tobacco leaves based on hyperspectral reflectance. Field Crops Research, 2013; 150: 108-114.

[4] De Pascale S, Maggio A, Orsini F, Barbieri G. Cultivar, soil type nitrogen source and irrigation regime as quality determinants of organically grown tomatoes. Scientia Horticulturae, 2016; 199: 88-94.

[5] Sritontip C, Khaosumain Y, Changjeraja S. Different nitrogen concentrations affecting chlorophyll and dry matter distribution in sand-cultured longan trees. Acta Hortic, 2014; 1024: 227-233.

[6] Oroka F O. Mulching effects and nitrogen application on the performance of Zea mays L: crop growth and nutrient accumulation. International Letters of Natural Sciences, 2016; 51: 36-42.

[7] Guzman J G, Godsey C B, Pierzynski G M, Whitney D A, Lamond R E. Effects of tillage and nitrogen management on soil chemical and physical properties after 23 years of continuous sorghum. Soil and Tillage Research, 2006; 91(1-2): 199-206.

[8] Horchani F, Hajri R, Aschi-Smiti S. Effect of ammonium or nitrate nutrition on photosynthesis, growth, and nitrogen assimilation in tomato plants. Journal of Plant Nutrition and Soil Science, 2010; 173(4): 610-617.

[9] Fatima T, Teasdale J R, Bunce J, Mattoo A K. Tomato response to legume cover crop and nitrogen: differing enhancement patterns of fruit yield, photosynthesis and gene expression. Functional Plant Biology, 2012; 39(3): 246-254.

[10] Kruse J, Hänsch R, Mendel R R, Rennenberg H. The role of root nitrate reduction in the systemic control of biomass partitioning between leaves and roots in accordance to the $\mathrm{C} / \mathrm{N}$-status of tobacco plants. Plant and Soil, 2010; 332: 387-403.

[11] Prause J Fernandez Lopez C. Litter decomposition and lignin/cellulose and lignin/total nitrogen rates of leaves in four species of the Argentine Subtropical forest. Agrochimica, 2007; 51(6): 294-300.

[12] Korus K, Conley M E, Paparozzi E T. Qualitative and quantitative analysis of soluble sugar content in leaves of hydroponically grown Swedish ivy at varying periods of nitrogen deficiency and subsequent re-greening. HortScience, 2008; 1285.

[13] Barickman T C, Kopsell D A, Sams C E. Abscisic acid improves tomato fruit quality by increasing soluble sugar concentrations. Journal of Plant Nutrition, 2017; 40(7): 964-973.

[14] Baxter C J, Carrari F, Bauke A, Overy S, Hill S A, Quick P W, et al. Fruit carbohydrate metabolism in an introgression line of tomato with increased fruit soluble solids. Plant and Cell Physiology, 2005; 46(3): 425-437.

[15] Taiz L Zeiger E, Møller I M, Murphy A. Plant physiology and development. Sinauer Associates, 2015.

[16] Zhao D L, Reddy K R, Kakani V G, Reddy V R. Nitrogen deficiency effects on plant growth, leaf photosynthesis, and hyperspectral reflectance properties of sorghum. European Journal of Agronomy, 2005; 22(4): 391-403.

[17] Shi.J Y, Zou X B, Zhao J W, Wang K L, Chen Z W, Huang X W, et al Nondestructive diagnostics of nitrogen deficiency by cucumber leaf chlorophyll distribution map based on near infrared hyperspectral imaging. Scientia Horticulturae, 2012; 138: 190-197.

[18] Sun J, Wang Y, Wu X H, Zhang X D. The nitrogen quantitative model based on hyperspectral image of tomato leaf. Advanced Materials 
Research, Trans, 2012; 466-467: 191-195.

[19] Liu Y L, Lyu Q, He S L, Yi S L, Liu X F, Xie R J, et al. Prediction of nitrogen and phosphorus contents in citrus leaves based on hyperspectral imaging. Int J Agric \& Biol Eng, 2015; 8(2): 80-88.

[20] Zhu Y, Wang W, Yao X. Estimating leaf nitrogen concentration (LNC) of cereal crops with hyperspectral data. In: Thenkabail P S (Ed.), Lyon J G (Ed.). Hyperspectral Remote Sensing of Vegetation. Boca Raton: CRC Press, 2012; pp.187-206.

[21] Sun J, Shi S, Gong W, Yang J, Du L, Song S L, et al. Evaluation of hyperspectral LiDAR for monitoring rice leaf nitrogen by comparison with multispectral LiDAR and passive spectrometer. Sci Rep, 2017; 7: 40362.

[22] Clevers J G P W, Kooistra L. Using hyperspectral remote sensing data for retrieving canopy chlorophyll and nitrogen content. IEEE Journal of Selected Topics in Applied Earth Observations and Remote Sensing, 2012; 5(2): 574-583.

[23] Song X, Xu D Y, He L, Feng W, Wang Y H, Wang Z J, et al. Using multi-angle hyperspectral data to monitor canopy leaf nitrogen content of wheat. Precision Agriculture, 2016; 17: 721-736.

[24] Zhang C, Liu F, Kong W W, Cui C, He Y, Zhou W J. Estimation and visualization of soluble sugar content in oilseed rape leaves using hyperspectral imaging. Transaction of the ASABE, 2016; 59(6): 1499-1505.

[25] Xu X G, Gu X H, Song X Y, Xu B, Yu H Y, Yang G J, et al. Assessing the ratio of leaf carbon to nitrogen in winter wheat and spring barley based on hyperspectral data. In: Proc. SPIE 9998, Remote Sensing for Agriculture, Ecosystems, and Hydrology XVIII. Edinburgh: SPIE, 2016. 999810.

[26] Shi R H, Niu Z, Zhuang D F. Feasibility of estimating leaf C/N ratio with hyperspectral remote sensing data. Remote Sensing Technology and Application, 2003; 18(2): 76-80. (in Chinese)

[27] Chen H B, Fan X H, Han Z G. A review on remote sensing from POLDER multispectral, multidirectional and polarized measurements. Remote Sensing Technology and Application, 2006; 21(2): 83-92. (in Chinese)

[28] Raven P N, Jordan D L, Smith C E. Polarized directional reflectance from laurel and mullein leaves. Optical Engineering, 2002; 41(5): 1002-1012.

[29] Shibayama M, Sakamoto T, Kimura A. A multiband polarimetric imager for field crop survey:-Instrumentation and preliminary observations of heading-stage wheat canopies-. Plant Production Science, 2011; 14(1): 64-74.

[30] del Ŕ1o L F, Arwin H, Järrendahl K. Polarization of light reflected from Chrysina gloriosa under various illuminations. Materials Today: Proceedings, 2014; 1: 172-176.

[31] Maxwell D J, Partridge J C, Roberts N W, Boonham N, Foster G D. The effects of surface structure mutations in Arabidopsis thaliana on the polarization of reflections from virus-infected leaves. PloS One, 2017; 12(3): e0174014

[32] Pourreza A, Lee W S, Etxeberria E, Zhang Y. Identification of citrus Huanglongbing disease at the pre-symptomatic stage using polarized imaging technique. IFAC-PapersOnLine, 2016; 49(16): 110-115.

[33] Wu T X, Zhang L F, Cen Y, Huang C P, Sun X J, Zhao H Q, et al. Polarized spectral measurement and analysis of sedum spectabile boreau using a field imaging spectrometer system. IEEE Journal of Selected Topics in Applied Earth Observations and Remote Sensing, 2013; 6(2): 724-730.

[34] Tan S X, Kabir Khan A S M. Water stress detection of lilac leaves using a polarized laser. In: Proc. SPIE 9610, Remote Sensing and Modeling of Ecosystems for Sustainability XII. San Diego: SPIE, 2015. 96100M.

[35] Song K S, Zhang B, Zhao Y S, Wang Z M, Du J. Study of polarized reflectance of corn leaf and its relationship to laboratory measurements of bi-directional reflectance. Journal of Remote Sensing, 2007; (5): 632-640.

[36] Liao Q H, Zhao C J, Yang G J, Coburn C, Wang J H, Zhang D Y, et al. Estimation of leaf area index by using multi-angular hyperspectral imaging data based on the two-layer canopy reflectance model. Intelligent Automation \& Soft Computing, 2013; 19(3): 295-304.

[37] Lü Y F. Study of hyperspectral polarized reflectance of vegetation canopy at nadir viewing direction. Spectroscopy and Spectral Analysis, 2013; 33(4): 1028-1031.

[38] Jay S, Maupas F, Bendoula R, Gorretta N. Retrieving LAI, chlorophyl and nitrogen contents in sugar beet crops from multi-angular optical remote sensing: Comparison of vegetation indices and PROSAIL inversion for field phenotyping. Field Crops Research, 2017; 210: 33-46.

[39] Mao H P, Zhu W J, Liu H Y. Determination of nitrogen and potassium content in greenhouse tomato leaves using a new spectro-goniophotometer. Crop and Pasture Science, 2014; 65(9): 888-898

[40] Zhu W J, Mao H P, Li Q L, Liu H Y, Sun J, Zuo.Z Y, et al. Study on the polarized reflectance-hyperspectral information fusion technology of tomato leaves nutrient diagnoses. Spectroscopy and Spectral Analysis, 2014; 34(9): 2500-2505.

[41] Chen Y W, Zeng Q Y, Pan Y X, Zhao Y. A new method of military false target identification. Electronic Design Engineering, 2011; 19(16): 89-92. (in Chinese)

[42] Story D, Kacira M, Kubota C, Akoglu A, An L L. Lettuce calcium deficiency detection with machine vision computed plant features in controlled environments. Computers and electronics in agriculture, 2010; 74(2): 238-243.

[43] Vittayapadung S, Zhao J W, Quansheng C, and Chuaviroj R. Application of FT-NIR spectroscopy to the measurement of fruit firmness of" Fuji" apples. Maejo International Journal of Science and Technology, 2008; 2(1): 13-23

[44] Ouyang Q, Zhao J W, Chen Q S. Measurement of non-sugar solids content in Chinese rice wine using near infrared spectroscopy combined with an efficient characteristic variables selection algorithm. Spectrochim Acta A: Mol Biomol Spectrosc, 2015; 151: 280-285.

[45] Norgaard L, Saudland A, Wagner J, Nielsen J P, Munck L, Engelsen S B. Interval partial least-squares regression (iPLS): A comparative chemometric study with an example from near-infrared spectroscopy. Applied Spectroscopy, 2000; 54(3): 413-419.

[46] Tewari J C, Dixit V, Cho B K, Malik K A. Determination of origin and sugars of citrus fruits using genetic algorithm, correspondence analysis and partial least square combined with fiber optic NIR spectroscopy. Spectrochimica Acta Part A: Molecular and Biomolecular Spectroscopy, 2008; 71(3): 1119-1127.

[47] Ouyang Q, Zhao J W, Chen Q S. Instrumental intelligent test of food sensory quality as mimic of human panel test combining multiple cross-perception sensors and data fusion. Anal Chim Acta, 2014; 841: 68-76.

[48] Cortes C, Vapnik V. Support-vector networks. Mach Learn, 1995; 20: 273-297.

[49] Cybenko G. Approximation by superpositions of a sigmoidal function. Math. Control Signals Systems, 1989; 2: 303-314.

[50] Wang L, Cui N, Fan Y H, Miao Q, Qu B,,Li T L. Comparison of carbohydrate metabolism after anthesis in the leaves of two tomato types. Journal of Shenyang Agriculture University, 2012; 43(4): 482-485. (in Chinese)

[51] Li Y X, Li J H, He L L, Gong G Y, Li T L. The effect of N. P. K mixed application on yields and quality of tomato in solar greenhouse. China Vegetables, 1997; 4: 12-15.

[52] Story D, Kacira M, Kubota C, Akoglu A. Morphological and textural plant feature detection using machine vision for intelligent plant health, growth and quality monitoring. Acta Hortic, 2011; 893: 299-306.

[53] Vanderbilt V C, Grant L, Daughtry C S T. Polarization of light scattered by vegetation. Proceedings of the IEEE, 1985; 73(6): 1012-1024.

[54] Mao H P, Gao H Y, Zhang X D, Kumi F. Nondestructive measurement of total nitrogen in lettuce by integrating spectroscopy and computer vision. Scientia Horticulturae, 2015; 184: 1-7. 